Francesco Prada, MD*

Alessandro Perin, MD, PhD*

Alberto Martegani, MD $\ddagger$

Luca Aiani, MD $\ddagger$

Luigi Solbiati, MD§

Massimo Lamperti, MDף

Cecilia Casali, MD*

Federico Legnani, MD*

Luca Mattei, MD*

Andrea Saladino, MD*

Marco Saini, MD*

Francesco DiMeco, MD* $\ddagger \S \uparrow \|$

*Department of Neurosurgery, and IICU, Department of Neurosurgery, Fondazione IRCCS Istituto Neurologico Carlo Besta, Milano, Italy; $\ddagger$ Department of Radiology, Ospedale Valduce, Como, Italy; §Department of Radiology, Ospedale di Circolo, Busto Arsizio, Italy; ||Department of Neurological Surgery, Johns Hopkins Medical School, Baltimore, Maryland

\section{Correspondence:}

Francesco Prada, MD,

Fondazione IRCCS Istituto

Neurologico Carlo Besta,

Via Celoria 11, 20133,

Milano, Italy.

E-mail: francesco.prada@istituto-besta.it

Received, September 5, 2013

Accepted, January 13, 2014.

Published Online, March 6, 2014.

Copyright $\odot 2014$ by the

Congress of Neurological Surgeons.

\title{
Intraoperative Contrast-Enhanced Ultrasound for Brain Tumor Surgery
}

BACKGROUND: Contrast-enhanced ultrasound (CEUS) is a dynamic and continuous modality that offers a real-time, direct view of vascularization patterns and tissue resistance for many organs. Thanks to newer ultrasound contrast agents, CEUS has become a wellestablished, live-imaging technique in many contexts, but it has never been used extensively for brain imaging. The use of intraoperative CEUS (iCEUS) imaging in neurosurgery is limited.

OBJECTIVE: To provide the first dynamic and continuous iCEUS evaluation of a variety of brain lesions.

METHODS: We evaluated 71 patients undergoing iCEUS imaging in an off-label setting while being operated on for different brain lesions; iCEUS imaging was obtained before resecting each lesion, after intravenous injection of ultrasound contrast agent. A semiquantitative, offline interobserver analysis was performed to visualize each brain lesion and to characterize its perfusion features, correlated with histopathology.

RESULTS: In all cases, the brain lesion was visualized intraoperatively with iCEUS. The afferent and efferent blood vessels were identified, allowing evaluation of the time and features of the arterial and venous phases and facilitating the surgical strategy. iCEUS also proved to be useful in highlighting the lesion compared with standard B-mode imaging and showing its perfusion patterns. No adverse effects were observed.

CONCLUSION: Our study is the first large-scale implementation of iCEUS in neurosurgery as a dynamic and continuous real-time imaging tool for brain surgery and provides the first iCEUS characterization of different brain neoplasms. The ability of CEUS to highlight and characterize brain tumor will possibly provide the neurosurgeon with important information anytime during a surgical procedure.

KEY WORDS: Brain tumor, Contrast-enhanced ultrasound, Glioma, Intraoperative imaging, Tumor perfusion, Ultrasound

\begin{tabular}{l} 
Neurosurgery 74:542-552, $2014 \quad$ DOI: $10.1227 /$ NEU.0000000000000301 \\
\hline
\end{tabular}

www.neurosurgery-online.com

maging techniques play a pivotal role during surgical resection of brain lesions. They help

when planning a surgical strategy before the operation, and during surgery, they indicate tumor

ABBREVIATIONS: CE, contrast enhancement; CEUS, contrast-enhanced ultrasound; EFSUMB, European Federation of Societies for Ultrasound in Medicine and Biology; 5-ALA, 5-aminolevulinic acid; iCEUS, intraoperative contrast-enhanced ultrasound; UCA, ultrasound contrast agent; US, ultrasound

Supplemental digital content is available for this article. Direct URL citations appear in the printed text and are provided in the HTML and PDF versions of this article on the journal's Web site (www.neurosurgery-online.com). boundaries and the relationships of the tumor with nearby vital structures, thus enhancing precision, accuracy, and safety for the patients, allowing maximal resection. ${ }^{1}$

The use of ultrasound (US) during neurosurgical procedures is becoming more widespread. Multiple studies have shown that US is a valuable tool in tumor detection during surgery. ${ }^{2-6}$ Although the quality of the gray-scale US image before tumor resection is excellent, little information is gained in terms of perfusion of the lesion, even with the aid of Doppler US. ${ }^{4,7-10}$

Contrast-enhanced ultrasound (CEUS) is a relatively new technique that has become progressively more common because it allows, among other things, highlighting of neoplastic lesions 
compared with the baseline US. ${ }^{11}$ CEUS is defined as lowmechanical index real-time contrast-specific imaging. ${ }^{12}$

Contrast agents containing microbubbles have been found to give the highest contrast. ${ }^{13,14}$ Microbubbles consist of air or inert gas encapsulated in a layer of proteins or polymers. Microbubbles are typically $5 \mu \mathrm{m}$ in diameter and can therefore be transported into the smallest capillaries and across the lungs. The passage across the lungs allows imaging of the arterial system with a venous injection. The pharmacokinetic of the microbubbles is quite different from that of contrast agents used for computed tomography and MRI, which generally diffuse in the interstitial space. ${ }^{15-17}$

Second-generation US contrast agents (UCAs) are clinically safe and well tolerated, and CEUS is an established technique for many organs. ${ }^{18}$

Microbubbles hit by low-acoustic-power US waves start resonating at a specific frequency according to their diameter; therefore, they reflect US and produce US with specific harmonics. These are detected and elaborated by the transducer through contrast-specific algorithms (Contrast Tuned Imaging, Power Modulation, Pulse Inversion Harmonic Imaging, Contrast Pulse Sequencing), ${ }^{14,15}$ allowing a continuous, nondestructive US scanning. ${ }^{16,18}$ This method permits a real-time assessment of tumor contrast enhancement (CE), measurement of vascularity of focal lesions during the different dynamic phases, and analysis of tissue perfusion, as well as online evaluation of treatment efficacy. ${ }^{19,20}$ For this reason, CEUS seems to be an ideal method to be tested for intraoperative visualization of brain lesions.

Only a few attempts have been made to try to visualize brain lesions with CEUS. ${ }^{21-28}$ Moreover, all of the studies cited above were based on preoperative, bedside, transcranial studies, being hampered by the limitation of the bony acoustic window. Only 3 neurosurgical studies (using UCAs) have been performed intraoperatively but on very small cohorts of patients and with some major technical limitations. ${ }^{29-31}$ In fact, no guidelines exist on intraoperative CEUS (iCEUS) in neurosurgery.

The main aim of this study is to evaluate and describe different brain pathologies by means of iCEUS compared with preliminary baseline US and preoperative MRI. This technique, being dynamic and continuous, allows a real-time direct view of the vascularization and flow distribution patterns of different types of neurosurgical lesions.

\section{METHODS}

\section{Study Design and Patient Population}

We performed iCEUS in an off-label setting following the European Federation of Societies for Ultrasound in Medicine and Biology (EFSUMB) guidelines on CEUS. ${ }^{12,18,32}$ We evaluated patients undergoing craniotomy for brain tumor removal with image guidance using a last-generation US system equipped with Fusion Imaging for neuronavigation (Esaote MyLab with Virtual Navigator, Esaote, Italy). Patients were bearing tumorlike brain masses, which were deemed surgically resectable, in good general status (American Society of Anesthesiologists 1-III; Karnofsky Performance Scale > 70). All patients were thoroughly informed about the surgical procedure, and written consent was obtained.
Intraoperative qualitative analysis was performed comparing iCEUS with B-mode US imaging and preoperative MRIs and correlated to histopathology. Tumor CE was then evaluated in an offline setting following the EFSUMB criteria on CEUS, ${ }^{12,18,32}$ performing an interobserver, semiquantitative analysis as described below.

\section{Equipment and Contrast Agent}

For US-guided neuronavigation, we used a last-generation US system with a multifrequency (3-11 MHz) linear probe. The US system was equipped with Virtual Navigator software for Fusion Imaging, allowing real-time neuronavigation between preoperative MRI and real-time US. As a contrast agent, we used sulfur hexafluoride-filled lipidic microbubbles, a secondgeneration UCA (SonoVue, Bracco, Italy), injected intravenously as a bolus $(2.4 \mathrm{~mL}[5 \mathrm{mg} / \mathrm{mL}])$. iCEUS scanning was performed with the Contrast Tuned Imaging algorithm that allows real time angiosonography.

\section{iCEUS Procedure and Data Analysis}

We performed preoperative, MRI-based surgical planning with neuronavigation coupled to US using the Virtual Navigator. After bone flap removal, a3- to 11-MHz linear US navigated probe (LA 332; Esaote, Italy) was placed in a surgical sterile transparent plastic sheath (CIVCO), along with $5 \mathrm{~mL}$ US transducing gel. The probe was placed over the dura to acquire standard B-mode imaging scans. The lesion was identified on the 2 axes and then measured. The lesion was also localized with standard neuronavigation and matched with the corresponding MRI scans. iCEUS was performed with a linear probe using low-power insonation; the consequent harmonic signals were transduced with the Contrast Tuned Imaging algorithm. Before the microbubble contrast agent was injected, the lesion was visualized with lowmechanical index B-mode imaging, along with healthy brain tissue; the focus of US was positioned at the bottom of the lesion. The contrast agent was injected intravenously by the anesthesiologist as a bolus $(2.4 \mathrm{~mL}[5 \mathrm{mg} / \mathrm{mL}])$, followed by a flush of saline solution $(10 \mathrm{~mL})$. The timer was started after UCA injection, and perfusion dynamics was described starting from UCA arrival in major vessels. A digital cine clip was registered during baseline US scanning and after contrast agent bolus injection for 100 to 300 seconds (median, 120 seconds).

A first online intraoperative qualitative analysis was performed to assess tumor/normal parenchyma echogenic characteristics, tumor boundaries, and specific anatomic landmarks. All lesions were initially evaluated with B-mode imaging, and a morphological qualitative online intraoperative correlation between US imaging and preoperative MRI was performed on the basis of a US real-time neuronavigation system that fused intraoperative cerebral US with preoperative MRI. Lesions on B-mode imaging were defined as highly hyperechoic, mildly hyperechoic, or isohypoechoic compared with the surrounding normal brain parenchyma. Other lesion characteristics were its appearance (diffuse or circumscribed), homogeneity vs heterogeneity, presence of calcification, and cystic/ necrotic areas. Then, an online qualitative analysis of intratumor microbubbles distribution and its relationships to other anatomic landmarks was conducted. A semiquantitative offline interobserver analysis was then performed by the first author (F.P., a neurosurgeon/certified sonologist) and by 3 external independent radiologists (A.M., L.A., and L.S.), with extensive experience in CEUS, to evaluate the visualization of each lesion with iCEUS following the EFSUMB guidelines ${ }^{12}$ : timing (arterial and venous phase [time is given as range]), degree of CE (comparison with brain parenchyma), and contrast distribution (centripetal/centrifugal pattern, visibility of afferent/efferent vessels, intralesion vessels, cystic/ necrotic areas). All data obtained by both online and offline analyses were correlated with the histopathology of each lesion. 


\section{RESULTS}

We evaluated 71 patients (age range, 10-76 years; mean, 50 years) harboring different intracranial lesions (53 gliomas [16 low grade, 9 anaplastic, 28 glioblastoma], 4 meningiomas, 6 metastases, 2 ependymomas, 1 pituitary adenoma, 1 hemangioblastoma, 1 ganglioglioma, 1 central neurocytoma, 1 abscess, 1 radionecrosis; see Table 1 for further details).

We were able to visualize all lesions both on B-mode and then with the iCEUS technique, with a complete interobserver agreement on both intraoperative and postoperative analyses. Standard US B-mode and iCEUS imaging features for each case are summarized in Table 2 and Figure 1.

We did not observe any adverse event or side effect related to the administration of the contrast agent.

We performed a morphological qualitative interobserver online analysis using an US-based real-time neuronavigation system that fuses intraoperative cerebral US with preoperative MRI, simultaneously displaying and comparing US and MRI. In all cases, we had great accuracy of correlation between intraoperative US imaging and preoperative MRI, being able to visually identify, to compare the lesions, and to easily recognize fixed anatomic

\begin{tabular}{|c|c|c|}
\hline Cases, $\mathbf{n}$ & Diagnosis & $\begin{array}{c}\text { World Health } \\
\text { Organization } \\
\text { Grade }\end{array}$ \\
\hline \multicolumn{3}{|l|}{ Gliomas } \\
\hline 2 & $\begin{array}{l}\text { Pilocytic } \\
\text { astrocytoma }\end{array}$ & I \\
\hline 14 & Low-grade glioma & II \\
\hline 9 & Anaplastic glioma & III \\
\hline 28 & Glioblastoma & IV \\
\hline \multicolumn{3}{|c|}{ Meningiomas } \\
\hline 2 & $\begin{array}{l}\text { Transitional } \\
\text { meningioma }\end{array}$ & I \\
\hline 1 & $\begin{array}{l}\text { Atypical } \\
\text { meningioma }\end{array}$ & II \\
\hline 1 & $\begin{array}{l}\text { Anaplastic } \\
\text { meningioma }\end{array}$ & III \\
\hline \multicolumn{3}{|c|}{$\begin{array}{l}\text { Other central nervous } \\
\text { systems primary tumors }\end{array}$} \\
\hline 1 & Ganglioglioma & I \\
\hline 1 & Pituitary adenoma & I \\
\hline 1 & Hemangioblastoma & I \\
\hline 2 & Ependymoma & II \\
\hline 1 & $\begin{array}{l}\text { Central } \\
\text { neurocytoma }\end{array}$ & II \\
\hline \multicolumn{3}{|c|}{$\begin{array}{l}\text { Secondary brain lesions/ } \\
\text { other }\end{array}$} \\
\hline 4 & Lung metastasis & - \\
\hline 1 & Colon metastasis & - \\
\hline 1 & Kidney metastasis & - \\
\hline 1 & Abscess & - \\
\hline 1 & Radiation necrosis & - \\
\hline
\end{tabular}

structure. We had a good morphological correspondence between the 2 imaging modalities with good correlation and superimposition between the 2 data sets, with an error $<2 \mathrm{~mm}$, as shown in Figure 2.

On standard US B-mode imaging, glioblastoma ( $\mathrm{n}=28)$ appeared all hyperechoic with to brain parenchyma, with a heterogeneous appearance composed of multiple well-defined nodular areas and others with diffuse margins. Maximal diameter ranged from 3 to $7 \mathrm{~cm}$. All but 3 lesions had cystic/necrotic areas (18 large and 3 with microcystic areas). Anaplastic astrocytoma $(\mathrm{n}=9)$ ranged from 4 to $7 \mathrm{~cm}$ in diameter. All 3 appeared hyperechoic with a diffuse, dense texture, with some areas more hyperechoic compared with the rest of the lesion. No cystic/ necrotic areas were noted. Low-grade gliomas $(\mathrm{n}=16)$ appeared mildly hyperechoic compared with brain parenchyma. Maximum diameter ranged from 3 to $6 \mathrm{~cm}$. All lesions had a homogeneous texture with blurred margins at the brain/tumor interface except for 1 oligoastrocytoma that had a discrete appearance with clear border and the only case of ganglioglioma. Microcysts were visible in only 2 cases. For both high- and low-grade lesions, the tumor margins were blurred; the brain/tumor interface was not clearly visible everywhere and was indistinguishable from edematous brain parenchyma.

Meningiomas $(n=4)$, regardless of World Health Organization grade, appeared hyperechoic, with fine granular aspects resulting from microcystic areas. Maximum diameter ranged from 3 to $9 \mathrm{~cm}$. Grade I and II meningiomas were homogeneous, whereas anaplastic ones appeared more heterogeneous, with multiple, vast hypoechoic areas caused by necrotic degeneration. All lesions were clearly well demarcated from surrounding brain tissue, even in the edematous areas.

All cerebral metastases $(n=6)$ were markedly hyperechoic with a diffuse granular and heterogeneous aspect. Their diameters ranged from 3 to $6 \mathrm{~cm}$. All lesions presented cystic areas, varying in size and calcifications. All lesions were clearly well demarcated from brain parenchyma.

Ependymomas $(\mathrm{n}=2)$ appeared as discrete nodular masses with a diffuse, dense texture with some minute hyperechoic areas compared with the rest of the lesion, probably resulting from microcalcification. One measured $5 \mathrm{~cm}$ and the other $3 \mathrm{~cm}$ in diameter. They both had multiple peripheral satellite cystic areas.

One pituitary adenoma was homogeneously hyperechoic and had a discrete multilobular appearance with clearly demarcated borders. Its maximum diameter was $5 \mathrm{~cm}$.

One posterior fossa hemangioblastoma appeared as a nodular hyperechoic mass surrounding a hypoechoic, heterogeneous center with clear borders.

One frontal ganglioglioma was a round mass, hyperechoic compared with brain parenchyma, with well-demarcated borders and an hypo-echoic cyst in the context of the lesion.

One case of central neurocytoma was deeply located, mildly hyperechoic, and heterogeneous with a fine multilobular aspect. Margins were, at times, diffused within the brain parenchyma. It measured $7 \mathrm{~cm}$ in diameter. 


\begin{tabular}{|c|c|c|c|c|c|c|c|c|c|c|c|}
\hline TABLE 2. Results St & nmary $^{a}$ & & & & & & & & & & \\
\hline \multirow[b]{3}{*}{ Brain Lesion } & \multirow[b]{3}{*}{$\begin{array}{c}\text { Patients, } \\
\text { n }\end{array}$} & \multicolumn{10}{|c|}{ Ultrasound Imaging Features } \\
\hline & & \multicolumn{3}{|c|}{ B-Mode } & \multicolumn{7}{|c|}{ CEUS } \\
\hline & & Echogenicity & Appearance & $\begin{array}{c}\text { Cystic Areas } \\
\text { and/or } \\
\text { Necrosis }\end{array}$ & $\begin{array}{l}\text { Arterial } \\
\text { Phase, } \mathbf{s}\end{array}$ & $\begin{array}{l}\text { CEUS } \\
\text { Peak, } s\end{array}$ & $\begin{array}{l}\text { Venous } \\
\text { Phase, } s\end{array}$ & $\begin{array}{c}\text { CE } \\
\text { Degree }\end{array}$ & CE Pattern & $\begin{array}{l}\text { Afferent/ } \\
\text { Efferent } \\
\text { Vessels }\end{array}$ & $\begin{array}{c}\text { Intralesional } \\
\text { Vessels }\end{array}$ \\
\hline Low-grade glioma & 16 & $\begin{array}{l}\text { Iso- } \\
\text { hyperechoic }\end{array}$ & $\begin{array}{l}\text { Diffuse, } \\
\text { homogeneous }\end{array}$ & $\begin{array}{l}\text { Small/ } \\
\text { microcysts }\end{array}$ & 15 & 20 & 30 & Mild & $\begin{array}{l}\text { Diffuse, dotted, } \\
\text { homogeneous, } \\
\text { centrifugal, no } \\
\text { cysts }\end{array}$ & $\begin{array}{l}\text { Not } \\
\text { visible }\end{array}$ & None \\
\hline Anaplastic glioma & 9 & $\begin{array}{l}\text { Iso- } \\
\text { hyperechoic }\end{array}$ & $\begin{array}{l}\text { Diffuse, } \\
\text { homogeneous }\end{array}$ & $\begin{array}{l}\text { Small/ } \\
\text { microcysts }\end{array}$ & 10 & 15 & $20-25$ & $\begin{array}{l}\text { Mild/ } \\
\text { high }\end{array}$ & $\begin{array}{l}\text { Diffuse, dotted, } \\
\text { homogeneous, } \\
\text { centrifugal } \\
\text { microcysts }\end{array}$ & Visible & Small \\
\hline Glioblastoma & 28 & Hyperechoic & $\begin{array}{l}\text { Diffuse/ } \\
\text { circumscribed, } \\
\text { heterogeneous }\end{array}$ & $\begin{array}{l}\text { Large } \\
\text { necrotic } \\
\text { areas }\end{array}$ & $2-3$ & 5 & 10 & High & $\begin{array}{l}\text { Nodular, highly } \\
\text { heterogeneous, } \\
\text { centripetal necrotic } \\
\text { areas }\end{array}$ & Visible & Large \\
\hline Meningioma & 4 & Hyperechoic & Circumscribed & Microcysts & $5-10$ & 15 & 30 & High & $\begin{array}{l}\text { Nodular, } \\
\text { homogeneous/ } \\
\text { heterogeneous. } \\
\text { centripetal necrotic } \\
\text { areas }\end{array}$ & Visible & Small/large \\
\hline Ganglioglioma & 1 & Hyperechoic & $\begin{array}{l}\text { Circumscribed, } \\
\text { homogeneous }\end{array}$ & $\begin{array}{l}\text { Small/ } \\
\text { microcysts }\end{array}$ & $2-3$ & 5 & 10 & $\begin{array}{l}\text { Mild/ } \\
\text { high }\end{array}$ & $\begin{array}{l}\text { Nodular, } \\
\text { homogeneous } \\
\text { centripetal } \\
\text { microcysts/ } \\
\text { macrocysts }\end{array}$ & Visible & Small \\
\hline Ependymoma & 2 & Hyperechoic & $\begin{array}{l}\text { Circumscribed, } \\
\text { homogeneous }\end{array}$ & $\begin{array}{l}\text { Small/ } \\
\text { microcysts }\end{array}$ & 5 & $5-10$ & $20-25$ & High & $\begin{array}{l}\text { Nodular, } \\
\text { homogeneous } \\
\text { centripetal } \\
\text { microcysts/ } \\
\text { macrocysts }\end{array}$ & Visible & Small \\
\hline Ctr. neurocytoma & 1 & $\begin{array}{l}\text { Variably } \\
\text { hyperechoic }\end{array}$ & $\begin{array}{l}\text { Nodular, } \\
\text { heterogeneous }\end{array}$ & None & $15-20$ & 40 & 30 & Mild & $\begin{array}{l}\text { Nodular, } \\
\text { heterogeneous } \\
\text { centripetal } \\
\text { microcysts/ } \\
\text { macrocysts }\end{array}$ & None & None \\
\hline Pituitary adenoma & 1 & Hyperechoic & $\begin{array}{l}\text { Circumscribed, } \\
\text { homogeneous }\end{array}$ & Microcysts & 10 & 30 & $>40$ & $\begin{array}{l}\text { Mild/ } \\
\text { high }\end{array}$ & $\begin{array}{l}\text { Diffuse, nodular, } \\
\text { homogeneous, } \\
\text { centrifugal } \\
\text { microcysts }\end{array}$ & None & None \\
\hline Hemangioblastoma & 1 & $\begin{array}{l}\text { Variably } \\
\text { hyperechoic }\end{array}$ & $\begin{array}{l}\text { Circumscribed, } \\
\text { homogeneous }\end{array}$ & None & 5 & 10 & 30 & High & $\begin{array}{l}\text { Nodular, } \\
\text { homogeneous, } \\
\text { centrifugal } \\
\text { microcysts/ } \\
\text { macrocysts }\end{array}$ & Visible & Small/large \\
\hline
\end{tabular}




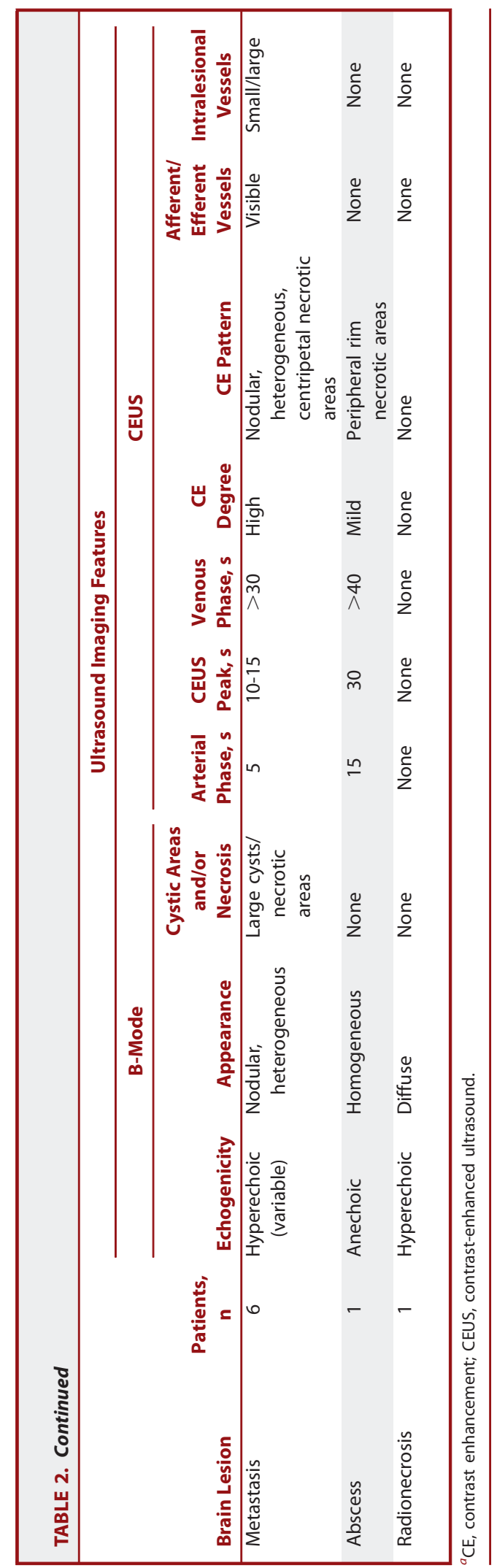

One case of cerebral abscess was superficial, with a thin hyperechoic capsule surrounding a large hypo-echoic center, measuring $5 \mathrm{~cm}$ in maximum diameter.

One case of radionecrosis had a homogeneous, diffuse, mildly hyperechoic texture with blurred margins at the brain/tumor interface.

Specific iCEUS patterns were observed in relation to the different pathological entities being tested. In our series, all neoplastic lesions showed CE, whereas 2 nonneoplastic lesions (1 abscess and 1 radionecrosis) did not.

Glioblastomas showed a rapid CE (20-30 seconds after UCA injection) marked by rapid arterial phase (2-3 seconds), very fast CE peak ( $3-5$ seconds), and chaotic transit of microbubbles within the lesion. The arterial supply was clearly visible, showing many macrovessels within the lesion and a typical peripheral enhancement that moved toward the inner areas of the lesion. The venous phase was rapid (5-10 seconds), and the venous drainage system was diffuse, with multiple medullary veins aiming toward the periventricular zone. (See Figure 3 and Video, Supplemental Digital Content 1, http://links.lww.com/NEU/A615, which is an intraoperative video obtained before the resection of a right parietal glioblastoma. The screen displays dual ultrasound imaging of the same field of view with low-mechanical index US B-mode imaging on the left-hand side and iCEUS imaging on the right hand side of the screen. Standard B-mode US allows identification of the region of interest while iCEUS is performed. On the left inferior corner is also visible a timer, which is manually started after UCA injection and permits the evaluation of the different vascular phases. Note how iCEUS is able to show the dynamic perfusion of the lesion, highlighting major feeding vessels, intralesion arteries, tumor mass, cystic/necrotic areas, and draining veins. iCEUS degree of enhancement of the lesion is compared with normal brain parenchyma CE.) $\mathrm{CE}$ was intense, with a persistent parenchymal phase compared with normal brain parenchyma and a heterogeneous CE pattern characterized by peripheral enhancement surrounding either highCE nodular areas or low/absent-CE, hypoperfused necrotic/cystic areas. Tumor borders were better defined after CE than in standard B-mode imaging.

Anaplastic astrocytoma appeared to have a slower arterial phase compared with glioblastomas (30-40 seconds after UCA injection; CE peak at 10-15 seconds after UCA arterial enhancement), with a venous phase at 20 to 25 seconds. Arterial supply was clearly visible with a centrifugal pattern and few macrovessels within the lesion. Venous drainage was not clearly identifiable. Anaplastic astrocytoma appeared to have a diffuse, progressive CE pattern, more homogeneous than the glioblastomas, reinforced during the parenchymal phase. Tumor borders are less visible after $\mathrm{CE}$ than in glioblastomas.

In the low-grade glioma group, the arterial phase had timing similar to that of anaplastic astrocytomas. Nevertheless, the CE peak was even more delayed (15-20 seconds) as well as the venous phase ( $>30$ seconds). The direct arterial supply and the venous drainage were not always clear. The arterial supply was centrifugal, and major vessels were not visible within the lesion. Astrocytomas were mildly hyperechoic after UCA injection compared with the brain parenchyma, and the tumor 


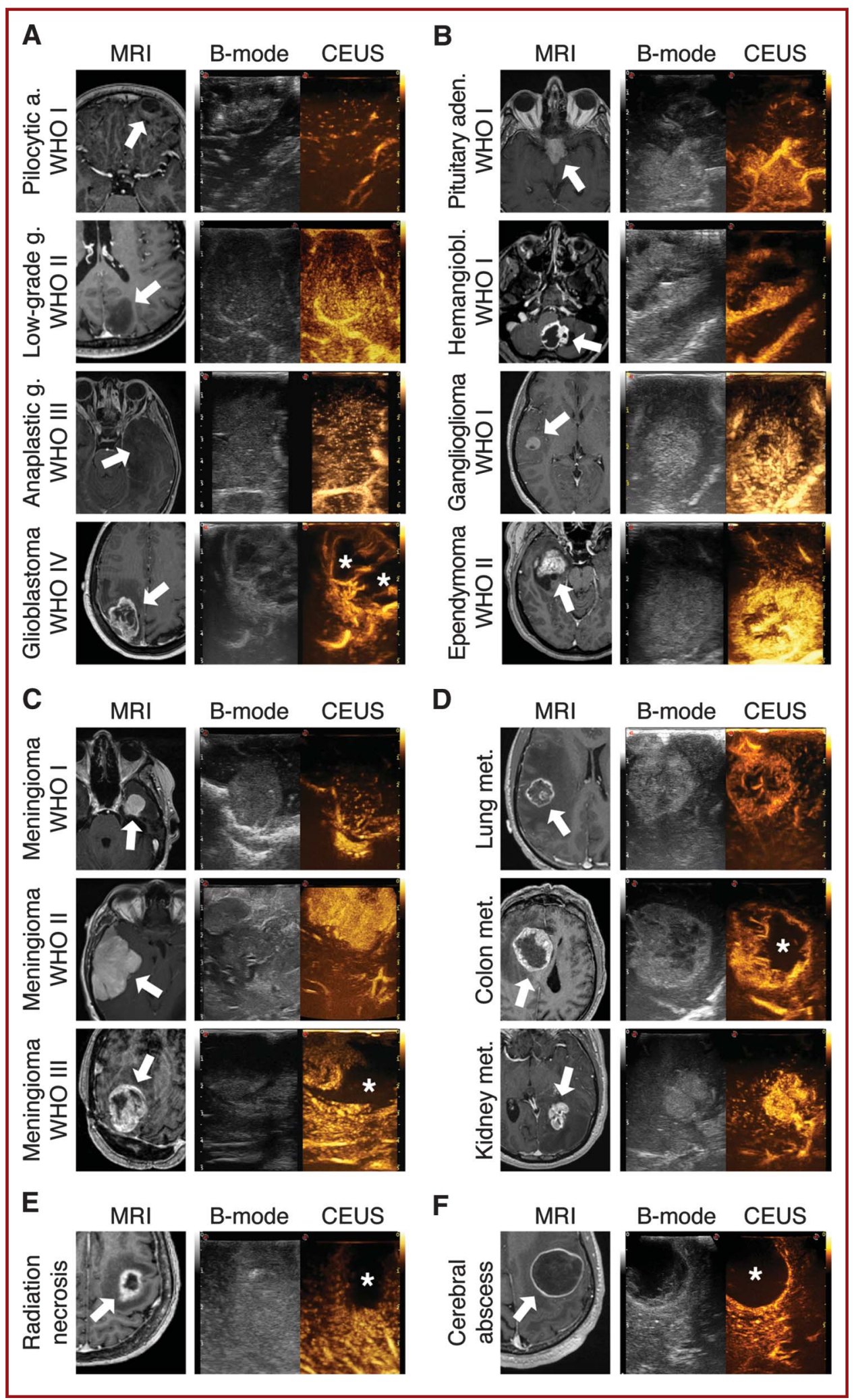


FIGURE 1. A comprehensive panel correlating magnetic resonance imaging (MRI) and ultrasound (US) appearance (both B-mode and contrast-enhanced US [CEUS]) of different brain lesions grouped by pathology (A, gliomas; $\mathbf{B}$, other tumors; C, meningiomas; D, metastases; $\mathbf{E}$, radiation necrosis; and $\mathbf{F}$, cerebral abscess). Every brain lesion is highlighted on a contrast-enhanced MRI (arrow). Note how preoperative MRI, B-mode, and CEUS appearances correlate (normal and pathological vessels look orange in CEUS; nonvascularized/cystic/necrotic areas are black [star]). a., astrocytoma; aden., adenoma; g., glioma; WHO, World Health Organization.

parenchymal phase was steady and uniform. The CE pattern was dotted and homogeneous, with only 2 cases with microcystic areas. In the 3 cases of oligodendroglioma, we observed features similar to those in anaplastic astrocytoma.

Meningiomas showed an intense and rapid CE (arterial phase, 20-30 seconds after UCA injection) with even higher CE peaks in higher grades. $\mathrm{CE}$ was centripetal with the major supply from the dural attachment. All grades displayed a dense and persistent parenchymal enhancement, being more homogeneous in grade I meningiomas. The venous phase was delayed $(>30$ seconds after UCA injection), and venous drainage was not clearly visible. Intralesional major vessels were visible only in grade II and III meningiomas, which also exhibited some hypodense/necrotic areas within the lesion. Tumor borders were clearly visible in all cases.

Cerebral metastasis showed a rapid CE (20-30 seconds after UCA injection), with a fast arterial phase (2-3 seconds) and CE peak (5-10 seconds). The arterial supply was centripetal, with many macrovessels within the lesion. CE was persistent with a delayed venous phase ( $>30$ seconds). The venous drainage system was not clearly visible. CE in metastases was very strong and intense with an irregular and heterogeneous CE pattern composed of high-contrast dense areas, macrovessels, and hypoperfused necrotic or nonperfused cystic areas. Tumor borders were clearly defined after CE.

Both ependymoma cases were characterized by a rapid arterial phase ( 5 seconds) and a very fast CE peak (5-10 seconds after UCA arrival). The arterial supply was clearly visible and centripetal, with many macrovessels well distinguishable within the lesion. The venous phase was rapid (20-25 seconds), and the venous drainage system was diffuse around the lesion. Ependymomas displayed an irregular and heterogeneous CE pattern with homogeneous areas alternating with hypoperfused, cystic areas.

The pituitary adenoma had a slow arterial phase (40-45 seconds after UCA injection) and a delayed CE peak (20 seconds after UCA arrival). The arterial supply and venous drainage were not visible, and the CE pattern was quite regular and homogeneous. No vessels were visible. The parenchymal phase was persistent with a slow venous phase ( $>40$ seconds).

One posterior fossa hemangioblastoma case displayed a rapid wash in phase (5-10 seconds after UCA arrival) and CE peak. Hemangioblastoma had an intense $\mathrm{CE}$ with a centripetal perfusion. The CE pattern showed a peripheral homogeneous parenchymal area surrounding a hypoperfused center (larger than what was visible on B-mode US only). No major vessels were visible within the lesion. The venous phase was delayed up to 30 seconds.

Ganglioglioma had a rapid arterial phase and CE peak (2-3 seconds after UCA arrival in arteries), with a homogeneous, bright CE pattern, few small vessels within the lesion, and a hypoechoic cystic area, superimposable to what was visible on B-mode imaging. The venous phase was rapid as well (10-15 seconds after arterial phase).

Our case of central neurocytoma showed a delayed arterial phase (30-40 seconds after UCA arrival) and CE peak. There was a mild $\mathrm{CE}$ with multiple homogeneous nodularity. No vessels were visible within the lesion, and the washout was delayed ( $>40$ seconds).

Finally, 1 case of cerebral abscess and 1 radionecrosis had no CE. The abscess had a thin, peripheral rimlike $\mathrm{CE}$ area surrounding a large necrotic center, whereas the radionecrosis remained completely without CE (being homogeneously hyperechoic on B-mode US).

\section{DISCUSSION}

In our study, we were able to directly visualize each of the 71 different brain lesions before resection with both the B-mode and iCEUS modalities and to surgically resect them under direct visualization with no adverse effects.

Our study shows that iCEUS is very useful in evaluating the location, defining the border, and depicting the vascularization and perfusion pattern of different brain tumors. Especially in tumors with ill-defined borders on B-mode US such as gliomas, iCEUS was very helpful in highlighting the lesion and its boundaries and possibly differentiating between tumor/edematous brain tissue.

We also demonstrate, although in a limited number of cases, the dynamic morphological and vascular pattern of cerebral tumor, which has only partially been shown before, demonstrating the ability of US and iCEUS to show rapid and dynamic events such as the arterial and venous phases of various lesions and their different pattern and degree of enhancement.

The evaluation of different parameters such as timing of the arterial and venous phases, peak, and intensity of $\mathrm{CE}$ and the presence of intralesion vessels or cystic/necrotic areas, will possibly lead, with its application in a higher number of cases, to the identification of specific iCEUS patterns for different cerebral lesions. This not only will contribute to enhancing our intraoperative knowledge in regard to tumor biology but also will lead to an iCEUS characterization of cerebral tumors, as it has been already established by the radiological community for other organs. ${ }^{12,16,18,35}$

The ability of iCEUS to highlight vascular structures made it easier for the surgeon to identify the vascular peduncles, giving further insight into the surgical strategy, facilitating vascular deafferentation of the lesion and then its surgical removal. After gross tumor removal, iCEUS might also be used to highlight tumor remnants, thus maximizing resection and avoiding neurological sequelae resulting from damaged healthy brain tissue. 

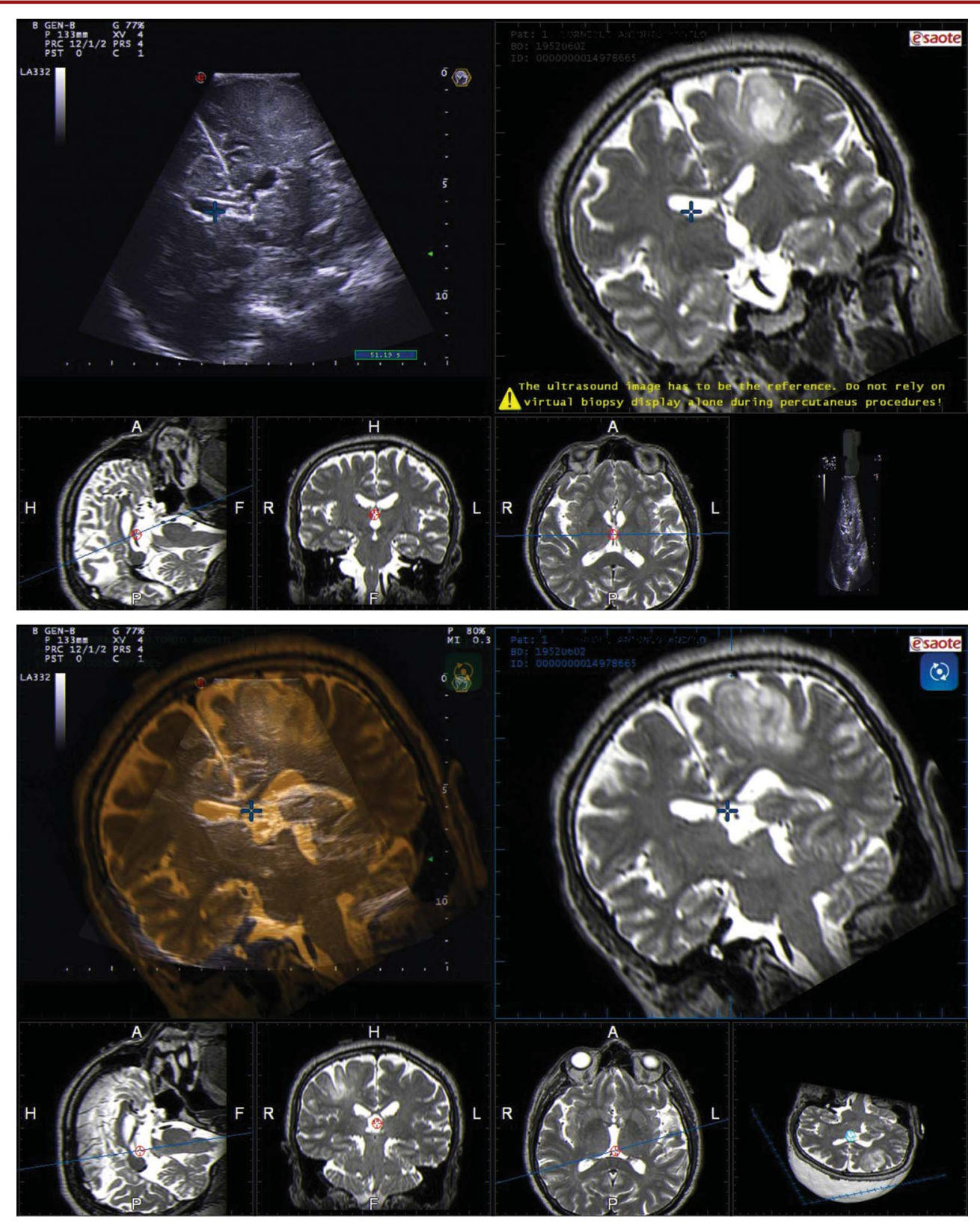

FIGURE 2. Virtual navigation unit screenshot used for intraoperative ultrasound (US) and preoperative magnetic resonance imaging (MRI) comparison, showing a left frontal mesial low-grade glioma (World Health Organization grade II). In the top panel, it is possible to visualize the MRI-based coronal visualization of the lesion in the right portion of the screen. The left portion of the screen shows the intraoperative US imaging, which is correlated to the navigated MRI. In the inferior portion of the screen, the different planes (sagittal, coronal, axial, plane of probe) are depicted. In the inferior panel, the 2 imaging modalities have been overlapped to evaluate the degree of correlation of the system and to evaluate and compare the different features of the lesion on both modalities.

Quite a few transcranial CEUS bedside studies have been performed on cerebrovascular patients to asses brain perfusion, extent of stroke, and response to stroke therapy ${ }^{24,36,37}$; on the other hand, only a few studies have investigated the role of iCEUS in highlighting cerebral tumors. ${ }^{21,22,27}$ In these studies, the authors showed the possibility of visualizing brain lesions with CEUS. Transcranial US is a well-established technique, but because of the cranial vault, it has a major limitation for brain tumor detection. Even 


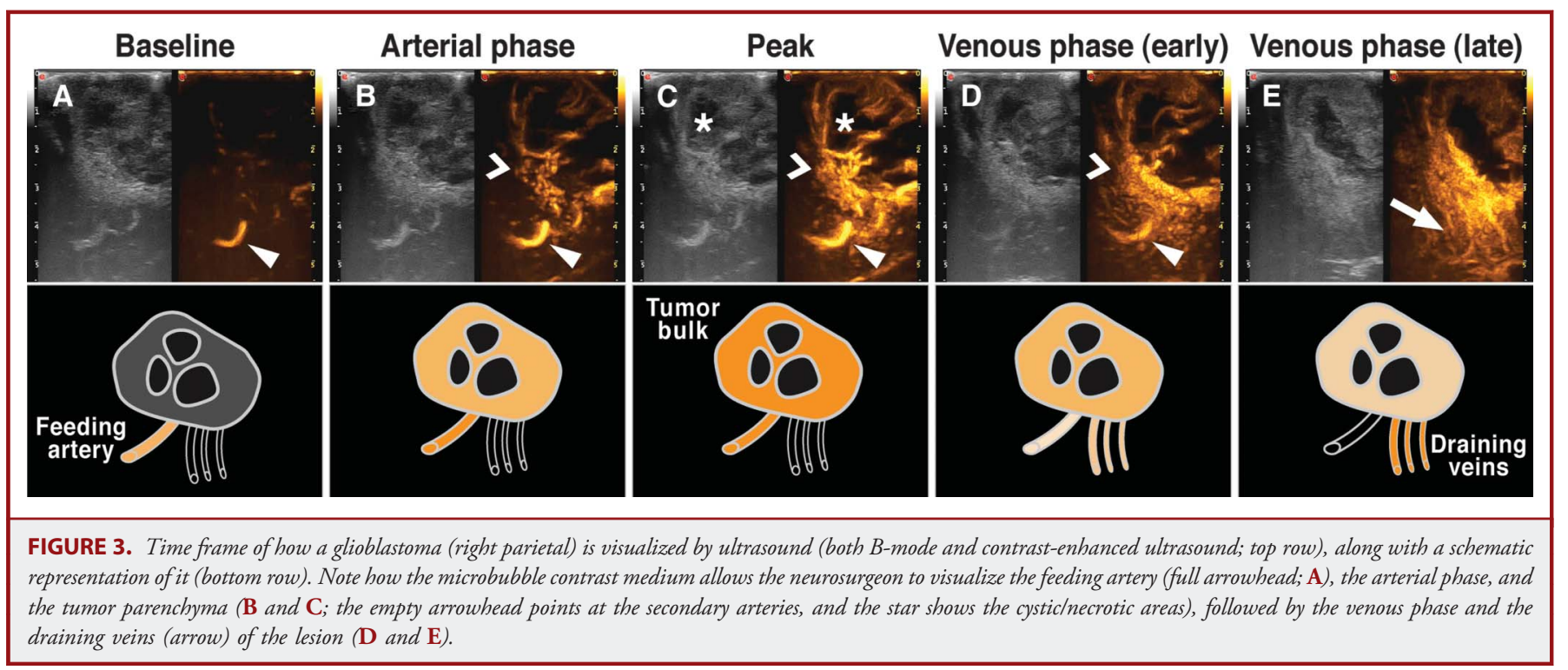

at the level of the temporal bone, despite its limited thickness, it is necessary to use a low-frequency insonation that does not allow high resolution and detailed evaluation of the brain parenchyma. Moreover, the use of a limited and fixed bone window does not allow the visualization of all kinds of lesions, and it is strongly dependent on the site of the lesion. ${ }^{22,23,38,39}$ Harrer et $\mathrm{al}^{22,23,39}$ by using preoperative CEUS, were able to distinguish some brain lesions from the brain parenchyma and to partially characterize different cerebral lesions. In another study, Vicenzini et $\mathrm{al}^{21}$ obtained time intensity/curves using dedicated software. However, in this series, the study population was small. In fact, time-intensity curves need very large cohorts to obtain a statistically significant result, as well as validation study for quantitative analysis. ${ }^{40}$ Nevertheless, these studies demonstrate the increasing interest in CEUS and brain tumors.

iCEUS has been undertaken in an intraoperative setting for brain tumor surgery only a few times, obtaining direct tumor visualization but with some limitations. ${ }^{29-31}$ Kanno et $\mathrm{al}^{29}$ analyzed 40 brain tumors using a first-generation contrast agent, obtaining only frame-by-frame imaging. Engelhardt et $\mathrm{al}^{30}$ made a step forward, performing iCEUS during brain tumor removal using a secondgeneration contrast agent with a specific algorithm and performing offline time-intensity curves, but on a very small and homogeneous cohort of patients (7 glioblastoma patients). Indeed, to provide statistically relevant results with time-intensity curves, they should be performed on a large sample size. ${ }^{41} \mathrm{He}$ et $\mathrm{al}^{31}$ intraoperatively evaluated 29 brain tumors ( 22 gliomas and 7 meningiomas) using iCEUS technique. They used a phased-array probe with low frequency and a vast view field (as used in transcranial Doppler), and the US imaging was performed in power Doppler modality instead of with a contrast-specific algorithm, dramatically reducing both US spatial resolution and definition.

Another study by Wang et $\mathrm{al}^{42}$ (only the abstract is available) evaluated the feasibility and value of intraoperative contrast- enhanced US in different pathological grades of glioma apparently without obtaining iCEUS characterization for these lesions.

Our findings also showed specific iCEUS features for each different kind of brain lesion, which might suggest a specific iCEUS pattern related to each different lesion. In glioblastomas, we found a heterogeneous CEUS pattern with rapid arterial and venous phases and high CE compared with brain parenchyma, and in all cases, it was always possible to visualize major arterial feeders. We also found almost invariably venous drainage through the periventricular medullary veins. The lesion was always well demarcated from brain parenchyma, and in some cases, iCEUS was able to show tumor remnants. Complete tumor demarcation was not always obtainable in anaplastic astrocytoma, even though a marked CE was clearly visible compared with brain parenchyma. Five anaplastic gliomas had been presurgically considered low-grade gliomas on the basis of MRI findings. In these cases, iCEUS showed a denser pattern with higher $\mathrm{CE}$ areas, probably expression of anaplastic foci within otherwise low-grade lesions.

Surprisingly, we found a subtle but clearly visible CE also in low-grade gliomas, which usually lack CE on MRI. In low-grade gliomas, iCEUS showed a scattered and dotted CE with slow vascular phases in all cases, even if borders were not always clearly distinguishable from healthy brain tissue.

For meningeal and other extra-axial vascular lesions such as hemangioblastoma, iCEUS showed a clear delineation of the lesion. Visualization of the arterial feeders and venous drainage is very helpful in planning the surgical strategy to progressively devascularize the lesion, avoiding profuse bleeding. In meningioma cases, however, complete lesion visualization could be impaired because of dural coagulation before CEUS scanning, thus reducing tumor perfusion. iCEUS confirmed the nonperfusion of avascular lesions such as abscess and radionecrosis, giving further assistance in differentiating these lesions from other lesions such as glioblastomas. 
Furthermore, US is a readily repeatable, dynamic, inexpensive procedure that can be performed any time for a potentially unlimited number of times during surgery. ${ }^{43}$ Other intraoperative imaging techniques such as intraoperative MRI and 5-aminolevulinic acid (5-ALA) are also able to highlight brain lesions but with some limitations compared with iCEUS. Intraoperative MRI, in addition to being expensive, requires the surgical procedure to stop during the acquisition phase, making the procedure considerably timeconsuming, whereas iCEUS imaging procedure takes a few minutes when properly conducted. For further accuracy and orientation, our US device is equipped with virtual navigation software that couples in real time the preoperative MRI with dynamic imaging provided with iCEUS. ${ }^{44}$ On the other hand, fluorescence-guided surgery such as that obtained with 5-ALA is an excellent method for intraoperative tumor visualization, but it can be adopted only in high-grade gliomas, whereas UCAs are, in our experience, able to visualize all kinds of brain lesions. Another major distinction depends on how tumor tissue is visualized. iCEUS shows on a screen a measurable section of the lesion before and at any given time during tumor resection, providing the surgeon with important information such as the on-site tumor morphology, the tumor relationships with anatomic landmark, and the thickness of tumor remnants during tissue resection. On the contrary, 5-ALA shows only the tumor surface. As a consequence, drawbacks in 5-ALA visualization might be due to neoplastic tissue hidden by healthy, nonfluorescent tissue. Furthermore, not all glioblastoma cells properly uptake 5-ALA, therefore reducing tumor visualization. ${ }^{45}$

\section{Limitations}

One major limitation of the CEUS technique is that individual imaging sections are applied with US, analyzing 1 portion of the lesion at a time. It is therefore mandatory to accurately scan the lesion in B-mode and color Doppler imaging before performing iCEUS to evaluate the more significant portion of the lesion and to obtain as much information as possible in regard to the timing. Offline analysis during surgery could be performed, allowing a more accurate interobserver analysis, even though it is difficult to share and transmit the whole set of information between all performers. However, a correlation between iCEUS and preoperative MRI (T1-weighted gadolinium) presents some major obstacles when comparing the degree of CE between the 2 techniques. In fact, microbubbles are purely intravascular, whereas MRI contrast agent is mainly interstitial. Furthermore, CEUS is a dynamic perfusion imaging, and MRI is static. ${ }^{33,34}$ We agree that this is a very interesting aspect, and further studies are needed in the future.

Care must also be taken in the preparation of UCA and its administration. Instructions for preparation must be followed accurately by the nurse anesthetist, and UCA injection has to be strictly synchronized with the start of the timer to achieve an accurate calculation of UCA transit time. To obviate such drawbacks, according to the EFSUMB guidelines, it is advisable that the surgeon who performs intraoperative US be trained for US and especially for CEUS, as in our case. ${ }^{12,18,39}$ Further studies are needed to evaluate the role of CEUS in tumor resection.

\section{CONCLUSION}

Our study is the first implementation in neurosurgery of iCEUS in a relatively large-scale setting. We found that iCEUS is a safe and fast intraoperative technique that completes and integrates the information obtained with standard B-mode and color Doppler imaging, providing dynamic and continuous real-time imaging and characterization of several brain lesions. Moreover, defining the paradigm of iCEUS adds valuable anatomic and biological information such as vascularization, microcirculation, and tissue perfusion dynamic, which will possibly provide further insights into the pathology of brain tumors, especially if integrated with other US modalities such as color Doppler and elastosonography.

Performing iCEUS before tumor removal might help the surgeon tailor the approach to the lesion, highlighting the lesion, clarifying between tumor and edematous brain tissue, and showing afferent and efferent vessels and hyperperfused areas, thus possibly modifying the intraoperative surgical strategy.

Further studies are needed to evaluate the role of iCEUS in highlighting tumor remnants after gross tumor removal, especially in high-grade lesions, thus maximizing resection and avoiding neurological sequelae.

In our experience, iCEUS has proven to be a simple and relatively inexpensive technique (especially compared with other intraoperative techniques such as 5-ALA and intraoperative MRI), and the expected results will potentially integrate scientific excellence and lead to better treatment for brain tumor patients, resulting in further understanding of and exploitation in the field of brain surgery.

\section{Disclosure}

The authors have no personal financial or institutional interest in any of the drugs, materials, or devices described in this article.

\section{REFERENCES}

1. Upadhyay UM, Golby AJ. Role of pre- and intraoperative imaging and neuronavigation in neurosurgery. Expert Rev Med Devices. 2008;5(1):65-73.

2. Sosna J, Barth MM, Kruskal JB, Kane RA. Intraoperative sonography for neurosurgery. J Ultrasound Med. 2005;24(12):1671-1682.

3. Unsgaard G, Gronningsaeter A, Ommedal S, Nagelhus Hernes TA. Brain operations guided by real-time two-dimensional ultrasound: new possibilities as a result of improved image quality. Neurosurgery. 2002;51(2):402-411; discussion 411-412.

4. Selbekk T, Jakola AS, Solheim O, et al. Ultrasound imaging in neurosurgery: approaches to minimize surgically induced image artefacts for improved resection control. Acta Neurochir (Wien). 2013;155(6):973-980.

5. Woydt M, Krone A, Becker G, Schmidt K, Roggendorf W, Roosen K. Correlation of intra-operative ultrasound with histopathologic findings after tumour resection in supratentorial gliomas: a method to improve gross total tumour resection. Acta Neurochir (Wien). 1996;138(12):1391-1398.

6. Woydt M, Vince GH, Krauss J, Krone A, Soerensen N, Roosen K. New ultrasound techniques and their application in neurosurgical intra-operative sonography. Neurol Res. 2001;23(7):697-705.

7. Lindseth F, Lovstakken L, Rygh OM, Tangen GA, Torp H, Unsgaard G. Blood flow imaging: an angle-independent ultrasound modality for intraoperative assessment of flow dynamics in neurovascular surgery. Neurosurgery. 2009;65 (suppl 6):149-157; discussion 157.

8. Mirzai S, Samii M. Current status and future challenges in cerebral blood flow mapping in intracranial tumors. Keio J Med. 2000;49(suppl 1):A16-A24. 
9. Becker G, Perez J, Krone A, et al. Transcranial color-coded real-time sonography in the evaluation of intracranial neoplasms and arteriovenous malformations. Neurosurgery. 1992;31(3):420-428.

10. Wang J, Liu X, Hou WH, et al. The relationship between intra-operative ultrasonography and pathological grade in cerebral glioma. J Int Med Res. 2008;36 (6):1426-1434.

11. Correas JM, Bridal L, Lesavre A, Méjean A, Claudon M, Hélénon O. Ultrasound contrast agents: properties, principles of action, tolerance, and artifacts. Eur Radiol. 2001;11(8):1316-1328

12. Piscaglia F, Nolsøe C, Dietrich CF, et al. The EFSUMB guidelines and recommendations on the clinical practice of contrast enhanced ultrasound (CEUS): update 2011 on non-hepatic applications. Ultraschall Med. 2012;33(1):33-59.

13. Claudon M, Cosgrove D, Albrecht T, et al. Guidelines and good clinical practice recommendations for contrast enhanced ultrasound (CEUS): update 2008. Ultraschall Med. 2008;29(1):28-44.

14. Mostbeck G. "Hot topics" in contrast-enhanced ultrasound (CEUS): introduction. Ultraschall Med. 2012;33(suppl 1):S1-S2.

15. Gibbs V, Cole D, Sassano A. Ultrasound Physics and Technology. Edinburgh, UK: Elsevier Health Sciences; 2009:111-119.

16. Quaia E. Assessment of tissue perfusion by contrast-enhanced ultrasound. Eur Radiol. 2011;21(3):604-615.

17. Martegani A, Aiani L, Borghi C. The use of contrast-enhanced ultrasound in large vessels. Eur Radiol. 2004;14(suppl 8):P73-P86.

18. Sidhu PS, Choi BI, Nielsen MB. The EFSUMB guidelines on the non-hepatic clinical applications of contrast enhanced ultrasound (CEUS): a new dawn for the escalating use of this ubiquitous technique. Ultraschall Med. 2012;33(1):5-7.

19. Lassau N, Chami L, Chebil M, et al. Dynamic contrast-enhanced ultrasonography (DCE-US) and anti-angiogenic treatments. Discov Med. 2011;11(56):18-24.

20. Solbiati L, Ierace T, Tonolini M, Cova L. Guidance and monitoring of radiofrequency liver tumor ablation with contrast-enhanced ultrasound. Eur J Radiol. 2004;51(suppl):S19-S23.

21. Vicenzini E, Delfini R, Magri F, et al. Semiquantitative human cerebral perfusion assessment with ultrasound in brain space-occupying lesions: preliminary data. J Ultrasound Med. 2008;27(5):685-692.

22. Harrer JU, Möller-Hartmann W, Oertel MF, Klötzsch C. Perfusion imaging of high-grade gliomas: a comparison between contrast harmonic and magnetic resonance imaging. Technical note. J Neurosurg. 2004;101(4):700-703.

23. Harrer JU, Hornen S, Oertel MF, Stracke CP, Klötzsch C. Comparison of perfusion harmonic imaging and perfusion MR imaging for the assessment of microvascular characteristics in brain tumors. Ultraschall Med. 2008;29(1):45-52.

24. Seidel G, Meyer K. Harmonic imaging: a new method for the sonographic assessment of cerebral perfusion. Eur J Ultrasound. 2001;14(2-3):103-113.

25. Meairs S, Daffertshofer M, Neff W, Eschenfelder C, Hennerici M. Pulse-inversion contrast harmonic imaging: ultrasonographic assessment of cerebral perfusion. Lancet. 2000;355(9203):550-551.

26. Federlein J, Postert T, Meves S, Weber S, Przuntek H, Büttner T. Ultrasonic evaluation of pathological brain perfusion in acute stroke using second harmonic imaging. J Neurol Neurosurg Psychiatry. 2000;69(5):616-622.

27. Bogdahn U, Fröhlich T, Becker G, et al. Vascularization of primary central hervous system tumors: detection with contrast-enhanced transcranial color-coded real-time sonography. Radiology. 1994;192(1):141-148.

28. Becker G, Winkler J, Bogdahn U. Transcranial color-coded real time sonography in adults. Part 2: cerebral hemorrhage and tumors [in German]. Ultraschall Med. 1991;12(5):211-217

29. Kanno H, Ozawa Y, Sakata K, et al. Intraoperative power Doppler ultrasonography with contrast-enhancing agent for intracranial tumors. J Neurosurg. 2005;102(2):295-301.

30. Engelhardt M, Hansen C, Eyding J, et al. Feasibility of contrast-enhanced sonography during resection of cerebral tumours: initial results of a prospective study. Ultrasound Med Biol. 2007;33(4):571-575.

31. He W, Jiang XQ, Wang $S$, et al. Intraoperative contrast-enhanced ultrasound for brain tumors. Clin Imaging. 2008;32(6):419-424

32. Bamber J, Cosgrove D, Dietrich CF, et al. EFSUMB guidelines and recommendations on the clinical use of ultrasound elastography, part 1: basic principles and technology. Ultraschall Med. 2013;34(2):169-184.
33. Whittle IR. Surgery for gliomas. Curr Opin Neurol. 2002;15(6):663-669.

34. Willems PW, Taphoorn MJ, Burger H, Berkelbach van der Sprenkel JW, Tulleken CA. Effectiveness of neuronavigation in resecting solitary intracerebral contrast-enhancing tumors: a randomized controlled trial. J Neurosurg. 2006;104(3):360-368.

35. Quaia E, Calliada F, Bertolotto M, et al. Characterization of focal liver lesions with contrast-specific US modes and a sulfur hexafluoride-filled microbubble contrast agent: diagnostic performance and confidence. Radiology. 2004;232(2):420-430.

36. Bartels E, Henning S, Wellmer A, Giraldo-Velásquez M, Kermer P. Evaluation of cerebral perfusion deficit in stroke patients using new transcranial contrast imaging CPS technology: preliminary results. Ultraschall Med. 2005;26(6):478-486.

37. Kern R, Perren F, Schoeneberger K, Gass A, Hennerici M, Meairs S. Ultrasound microbubble destruction imaging in acute middle cerebral artery stroke. Stroke. 2004;35(7):1665-1670

38. van Leyen $\mathrm{K}$, Klötzsch C, Harrer JU. Brain tumor imaging with transcranial sonography: state of the art and review of the literature. Ultraschall Med. 2011;32 (6): $572-581$.

39. Harrer JU, Mayfrank L, Mull M, Klötzsch C. Second harmonic imaging: a new ultrasound technique to assess human brain tumour perfusion. J Neurol Neurosurg Psychiatry. 2003;74(3):333-338

40. Leen E, Averkiou M, Arditi M, et al. Dynamic contrast enhanced ultrasound assessment of the vascular effects of novel therapeutics in early stage trials. Eur Radiol. 2012;22(7):1442-1450.

41. Giangregorio F, Bertone A, Fanigliulo L, et al. Predictive value of time-intensity curves obtained with contrast-enhanced ultrasonography (CEUS) in the follow-up of 30 patients with Crohn's disease. J Ultrasound. 2009;12(4):151-159.

42. Wang YQ, Yu SQ, Wang JS, et al. Application of intraoperative contrast-enhanced ultrasound in different pathological grades of glioma [in Chinese]. Zhonghua $Y i$ Xue Za Zhi. 2012;92(21):1495-1497.

43. Romanini L, Passamonti M, Aiani L, et al. Economic assessment of contrastenhanced ultrasonography for evaluation of focal liver lesions: a multicentre Italian experience. Eur Radiol. 2007;17(suppl 6):F99-F106.

44. Busse H, Schmitgen A, Trantakis C, Schober R, Kahn T, Moche M. Advanced approach for intraoperative MRI guidance and potential benefit for neurosurgica applications. J Magn Reson Imaging. 2006;24(1):140-151.

45. Stummer W, Novotny A, Stepp H, Goetz C, Bise K, Reulen HJ. Fluorescenceguided resection of glioblastoma multiforme by using 5 -aminolevulinic acidinduced porphyrins: a prospective study in 52 consecutive patients. J Neurosurg. 2000;93(6):1003-1013.

Supplemental digital content is available for this article. Direct URL citations appear in the printed text and are provided in the HTML and PDF versions of this article on the journal's Web site (www.neurosurgery-online.com).

\section{Acknowledgments}

The authors would like to thank Mrs Caroline King, DipArch, for her kind advice in revising the manuscript and Mr Luca Lodigiani for his technical support.

\section{COMMENT}

This is an important article dealing with contrast-enhanced ultrasound in brain tumor surgery. I believe that this technique should be further developed to offer neurosurgeons an extremely fast and rather costeffective imaging technology.

It has to be investigated whether the combination with intraoperative magnetic resonance imaging at the end of surgery or the standalone application can detect tumor remnants.

Martin Scholz Bochum, Germany 